\section{New understanding on the contribution of the central otolithic system to eye movement and skew deviation}

\begin{abstract}
The otolith organs consist of the utricle and saccule. The utricle mediates the utriculoocular reflex by detecting horizontal head translation and static head tilt. Skew deviation is a vertical strabismus caused by imbalance of the utriculo-ocular reflex pathway and is commonly caused by lesions in the brainstem or cerebellum. It is associated with abnormal utriculo-ocular reflexes including asymmetric reduction of the translational vestibulo-ocular and ocular counterroll responses. Skew deviation is also associated with head position-dependent changes in ocular torsion and vertical strabismus. The reduction in ocular torsion and vertical strabismus when changing from an upright to supine position in skew deviation allows us to devise a new bedside 'upright-supine test' to differentiate skew deviation from fourth nerve palsy and other causes of vertical strabismus.
\end{abstract}

Eye (2015) 29, 153-156; doi:10.1038/eye.2014.243; published online 17 October 2014

\section{Introduction}

Reflexive compensatory eye movements are generated to stabilize retinal images during head motion. The peripheral vestibular apparatus (or labyrinth) consists of the semicircular canals that detect head rotation and the otolith organs (utricle and saccule) that detect head translation and static head tilt. In this review, we will briefly discuss the anatomy and physiology of the vestibule-ocular reflex (VOR) pathways mediated by the otolith organs. We will then review recent evidence
AMF Wong

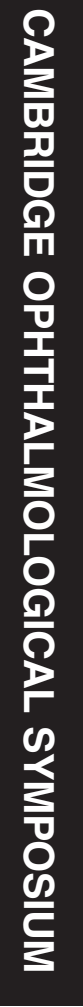

showing how imbalance of utricular input to the VOR pathway (utriculo-ocular reflex pathway) contributes to a pathological condition called skew deviation. Finally, we will discuss how understanding the pathophysiology of skew deviation leads to a new bedside clinical testthe upright-supine test-that can be used to differentiate skew deviation from other forms of vertical strabismus including fourth nerve palsy.

\section{Anatomy and physiology of the otoliths}

The membranous labyrinth is filled with endolymph. A portion of the utricle and saccule is thickened to form the macula that contains hair cells. The macula is covered with a gelatinous substance called otoconia. When the head is tilted or undergoes linear acceleration, the endolymph deforms the otoconia which bends the cilia on the hair cells and activate the vestibular nerve. ${ }^{1-3}$ The vestibular nerve consists of bipolar neurons with cell bodies located in the vestibular ganglion (Scarpa's ganglion). The vestibular nerve is divided into two divisions based on the portion of the end organ that they innervate. The superior division carries information arising from the macula of the utricle, the anterior part of the macula of the saccule, and the ampullae of the horizontal and anterior semicircular canals. The inferior division carries information from the posterior part of the macula of the saccule and the ampulla of the posterior canal. The vestibular nerve then joins the cochlear nerve to form the vestibulocochlear nerve (the eight cranial nerve), which traverses the cerebellopontine angle to enter the brainstem.

The otolith-ocular reflex originates in the maculae of the otolith organs, the utricle and Sciences, The Hospital for Sick Children, University of Toronto, and the Toronto Western Hospital, Toronto, Canada

Correspondence: AMF Wong, Department of Ophthalmology and Vision Sciences, The Hospital for Sick Children, 555 Ontario, Canada M5G 1X8 Tel: +1 4168137654 extn 202642;

Fax: +1 4168135159 .

E-mail: agnes.wong@ utoronto.ca

Received: 8 September 2014

Accepted in revised form:

12 September 2014

Published online: 17

October 2014

This work was presented at the 2014 Cambridge Ophthalmological Symposium.
Department of Ophthalmology and Vision

University Avenue, Toronto, 
saccule, which act as linear accelerometers. Hair cells on the maculae have a wide range of polarization vectors. The directions of the polarization vectors are reversed across a central zone in each macula called the striola. ${ }^{4,5}$ With the head in the upright position, the utricle lies roughly in an earth-horizontal plane, and is responsible for detecting horizontal translation and static head tilt (ie, utriculo-ocular reflex). The saccule, on the other hand, lies roughly in an earth-vertical plane and is responsible for detecting vertical translation and static head tilt.

The utriculo-ocular reflex pathway is disynaptic, similar to the canal-ocular pathway. Primary afferents arising from the utricle project to second-order neurons in the vestibular nuclei, which then carry signals via the medial longitudinal fasciculus to the oculomotor and trochlear nuclei. ${ }^{6,7}$ Polysynaptic pathways also have a critical role in mediating the utriculo-ocular reflex. ${ }^{8-10}$ Signals from the lateral utricle ipsilateral to the direction of head translation are transmitted to the ipsilateral vestibular nucleus, via an extensive network of projections within the cerebellum (including the nodulus, ventral uvula, fastigial nucleus, anterior vermis, and flocculus/ventral paraflocculus). Polysynaptic pathways are also responsible for generating ocular counterroll movements during static head tilt. ${ }^{10}$

\section{Skew deviation}

Skew deviation is a vertical strabismus caused by a supranuclear lesion, commonly in the brainstem or cerebellum. ${ }^{11,12}$ Abnormal ocular torsion, pathologic head tilt and abnormal tilt of the subjective visual vertical may be associated, constituting the ocular tilt reaction. ${ }^{13,14}$ Skew deviation has been attributed to asymmetric disruption of the utriculo-ocular reflex as a result of imbalance of utricular projections to ocular motor neurons (disynaptically through the brainstem or polysynaptically through the cerebellum); ${ }^{14,15}$ however, direct evidence of abnormal utricular functions has been lacking until we began to investigate its pathophysiology several years ago. Because the utricular macula lies roughly in the horizontal plane when the head is in the upright position, the utricle normally mediates the translational vestibulo-ocular reflex (LVOR) by detecting head translation in the horizontal plane (fore-aft and side-to-side), as well as the ocular counterroll reflex (OCR) by detecting static tilt of the head with respect to gravity. We hypothesized that if skew deviation is indeed caused by imbalance of utriculo-ocular reflex pathway, then: (1) the LVOR would be abnormal; (2) the OCR would be abnormal; and (3) the abnormal torsion and vertical strabismus seen in skew deviation would be head position-dependent.
To test whether the LVOR is abnormal in skew deviation, we constructed a head-sled device that allowed us to deliver transient, high-acceleration, 'head heave' impulses to the head along the interaural axis while eye movements were being measured using the magnetic scleral search coil technique. ${ }^{16}$ We validated our methodology first by showing that patients with documented otolith diseases exhibited reduction in their LVOR responses. ${ }^{17}$ We then applied the head-heave test to six patients with skew deviation and 10 normal subjects. Skew deviation was diagnosed when the 'three-step test' was negative in the presence of other neurologic symptoms and signs, as well as MRI confirmed brainstem or cerebellar lesion. We found that in patients with skew deviation, LVOR sensitivity gain (peak rotational eye velocity to peak linear head velocity) and velocity gain (peak actual-to-ideal rotational eye velocities) were reduced by $56-62 \%$ as compared with normal controls. In addition, the reduction was asymmetric in both eyes-it differed between eyes by $37-143 \%$ for sensitivity gain and by $36-94 \%$ for velocity gains. These findings provided the first evidence that skew deviation is associated with asymmetric reduction of LVOR response, and supported our first hypothesis that imbalance in the utriculo-ocular reflex pathway is a mechanism of skew deviation. ${ }^{18}$

Previous studies found that OCR gain varies considerably across healthy subjects, and this variability may result from using visual targets of different complexities and viewing distances. To test whether OCR is abnormal in skew deviation, we first sought to clarify the effects of age and target characteristic on OCR gain in a normal population across the age span $(n=60)$. We found that OCR gain is dependent on age, viewing distances, and target complexity, ${ }^{19}$ as well as whether the target is head fixed or earth fixed. ${ }^{20}$ Using an earth-fixed complex target at a distance of $1 \mathrm{~m}$ that has been shown to elicit higher gain in normal subjects, we recorded the eye movements of 18 patients with skew deviation and 18 normal participants by scleral search coil. ${ }^{21}$ We found that mean OCR gain was reduced by $45 \%$ in patients as compared with normal controls, and that OCR gains were asymmetric between eyes and between torsional directions in $90 \%$ of patients. Our findings that patients with skew deviation had abnormally low and asymmetric OCR gain provided support to our second hypothesis that imbalance in the utriculo-ocular reflex pathway is a mechanism of skew deviation. ${ }^{22}$

Because the otoliths normally detect static tilt of the head, we postulated that if skew deviation is caused by an imbalance of the utriculo-ocular reflex, the abnormal torsion and vertical strabismus would be head position-dependent. To test our hypothesis, we recruited 10 patients with skew deviation, 14 patients with 
unilateral peripheral cranial fourth nerve palsy, and 12 healthy subjects. Ocular torsion was measured by the double Maddox rods and vertical strabismus was measured by the prism and alternate cover test with subjects first in the upright position and then in the supine position. We found that the abnormal torsion and vertical misalignment in skew deviation were indeed head position-dependent-they reduced significantly when patients changed from an upright to supine position. This was in contrast to patients with fourth nerve palsy and normal subjects, whose torsion and vertical deviation remained unchanged in different head positions. Torsion was decreased by $83 \%$ from upright to supine in skew deviation, as compared with $2 \%$ in fourth nerve palsy, and $6 \%$ in healthy controls (Figure 1). Similarly, vertical strabismus was decreased by $74 \%$ from upright to supine in skew deviation (Figure 2). Our findings supported our third hypothesis and provided further evidence that abnormal utriculo-ocular reflex is a mechanism of skew deviation. ${ }^{23}$ The physiological basis of head position-dependent changes in torsion and vertical strabismus is most likely related to a change in orientation of the utricles from earth-horizontal in the upright position to earth-vertical in the supine position. This new orientation of the utricles may lead to a saturation (ceiling effect) or reduction (floor effect) in the overall activities of the utriculo-ocular reflex, such that

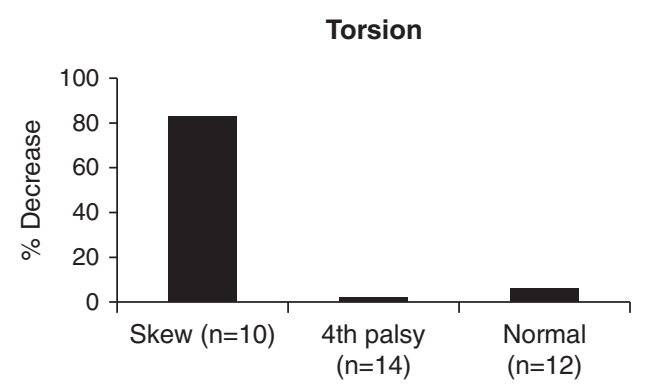

Figure 1 Percentage decrease in torsion from upright to supine position in patients with skew deviation, fourth nerve palsy, and normal controls.

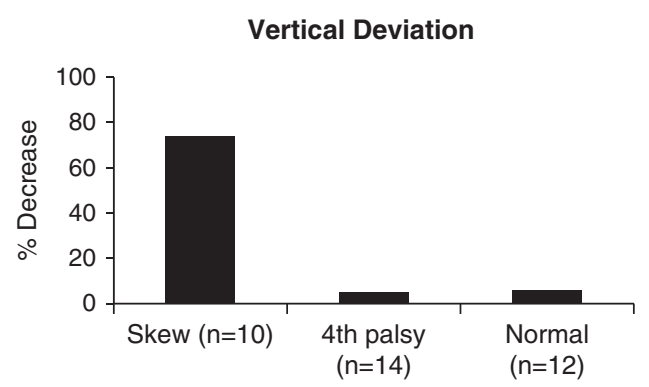

Figure 2 Percentage decrease in vertical strabismus from upright to supine position in patients with skew deviation, fourth nerve palsy, and normal controls. any asymmetry of the reflex is minimized. This, in turn, may lead to a reduction in torsion and vertical strabismus in skew deviation. In contrast, in fourth nerve palsy, the utriculo-ocular reflex pathway is intact; thus there is no change in torsion or vertical deviation in different head positions. In fact, the contralateral head tilt commonly seen in patients with fourth nerve palsy is a compensatory mechanism that utilizes the normal utriculo-ocular reflex to minimize the magnitude of vertical diplopia. This finding also points to an important caveat of using the Bielschowsky's head tilt test (the third step) to diagnose fourth nerve palsy-it is only effective and should only be performed in the upright position.

Because skew deviation can sometimes mimic fourth nerve palsy when the 'three-step test' is positive, differentiate between the two conditions may pose a diagnostic challenge to clinicians. Based on the head position-dependent changes we observed, ${ }^{23}$ we sought to devise a simple, new bedside test that will help clinicians to differentiate skew deviation from other causes of vertical strabismus including fourth nerve palsy. To determine the sensitivity and specificity of this new upright-supine test, we recruited 25 patients with skew deviation, 58 with unilateral fourth nerve palsy, 14 with restrictive causes (eg, thyroid eye disease, orbital trauma, Brown syndrome, and scleral buckle), 28 with other causes of vertical strabismus (eg, myasthenia gravis, childhood strabismus, and peripheral third nerve palsy), and 20 healthy controls. Their vertical strabismus was measured by the prism and alternate cover test using a near target at $1 / 3 \mathrm{~m}$ in upright and supine. A positive test was defined as a vertical strabismus that decreased by $50 \%$ or more from the upright to supine position. We found that the upright-supine test was positive in 20 of 25 patients with skew deviation, giving a sensitivity of $80 \%$. It was negative in all patients with other causes of vertical strabismus and normal controls, giving a specificity of $100 \%$ (Table 1). We concluded that the upright-supine test could be added as a fourth step after the 3-step test to differentiate skew deviation from other causes of vertical strabismus including fourth nerve palsy. If the result is positive, neuroimaging should be strongly considered. ${ }^{24}$

In conclusion, skew deviation is a vertical strabismus caused by imbalance of the utriculo-ocular reflex pathway in the brainstem or cerebellum. It is associated with abnormal utriculo-ocular reflexes, as evidenced by: (1) asymmetric reduction in LVOR responses; (2) asymmetric reduction in OCR responses; and (3) head position-dependent changes in torsion and vertical misalignment. Understanding the anatomy and physiology of the central otolithic pathway not only allows us to gain insight into the pathophysiologic mechanism of skew deviation, it also allows us to devise 
Table 1 Upright-supine test results in patients with skew deviaiton and other causes of vertical strabimsus

\begin{tabular}{lcc}
\hline & $\begin{array}{c}\text { Test positive } \\
(\geq 50 \% \text { decrease })\end{array}$ & $\begin{array}{c}\text { Test negative } \\
(<50 \% \text { decrease })\end{array}$ \\
\hline $\begin{array}{l}\text { Skew deviation }(n=25) \\
\text { Vertical strabismus other }\end{array}$ & 20 & 5 \\
than skew $(n=100)$ & 0 & 100 \\
\hline
\end{tabular}

Sensitivity $=20 / 25=80 \%$.

Specificity $=100 / 100=100 \%$.

a new clinical test to differentiate skew deviation from fourth nerve palsy and other causes of vertical strabismus.

\section{Conflict of interest}

The author declares no conflict of interest.

\section{Acknowledgements}

Supported by Grants MOP 152588 and MOP 57853 from the Canadian Institutes of Health Research, and the Department of Ophthalmology and Vision Science at The Hospital for Sick Children.

\section{References}

1 Lysakowski A, Goldberg JM. Morphophysiology of the vestibular periphery. In: Highstein SM, Fay RR, Popper AN eds. The Vestibular System. Springer-Verlag: New York, 2003; 57-152.

2 Wong AMF. Eye Movement Disorders. Oxford University Press: New York, 2008.

3 Sharpe JA, Wong AMF. Anatomy and physiology of ocular motor systems. In: Miller NR, Newman NJ, Biousse V, Kerrison JB eds. Walsh and Hoyt's Clinical NeuroOphthalmology Vol 16th ed. Lippincott Williams \& Wilkins: Philadelphia, Pennsylvania, 2005; 809-885.

4 Cohen B, Raphan T. The physiology of the vestibuloocular reflex (VOR). In: Highstein SM, Fay RR, Popper AN eds. The Vestibular System. Springer-Verlag: New York, 2003; 235-285.

5 Lindeman HH. Studies on the morphology of the sensory regions of the vestibular apparatus. Ergeb Anat Entwicklungsgesch 1969; 42: 1-113.

6 Uchino Y, Ikarashi K, Sasaki H, Endo K, Imagawa M, Isu N. Monosynaptic and disynaptic connections in the utriculoocular reflex arc of the cat. J Neurophysiol 1994; 71: 950-958.

7 Uchino Y, Sasaki M, Sato H, Imagawa M, Suwa H, Isu N. Utriculoocular reflex arc of the cat. J Neurophysiol 1996; 76: 1896-1903.
8 Wiest G, Tian JR, Baloh RW, Crane BT, Demer JL. Otolith function in cerebellar ataxia due to mutations in the calcium channel gene CACNA1A. Brain 2001; 124: 2407-2416.

9 Newlands SD, Vrabec JT, Purcell IM, Stewart CM, Zimmerman BE, Perachio AA. Central projections of the saccular and utricular nerves in macaques. J Comp Neurol 2003; 466: 31-47.

10 Angelaki DE. Eyes on target: what neurons must do for the vestibuloocular reflex during linear motion. J Neurophysiol 2004; 92: 20-35.

11 Brandt T, Dieterich M. Skew deviation with ocular torsion: a vestibular brainstem sign of topographic diagnostic value. Ann Neurol 1993; 33: 528-534.

12 Wong AMF, Sharpe JA. Cerebellar skew deviation and the torsional vestibuloocular reflex. Neurology 2005; 65: 412-419.

13 Halmagyi GM, Gresty MA, Gibson WPR. Ocular tilt reaction with peripheral vestibular lesion. Ann Neurol 1979; 6: 80-83.

14 Rabinovitch HE, Sharpe JA, Sylvester TO. The ocular tilt reaction. A paroxysmal dyskinesia associated with elliptical nystagmus. Arch Ophthalmol 1977; 95: 1395-1398.

15 Brodsky MC, Donahue SP, Vaphiades M, Brandt T. Skew deviation revisited. Surv Ophthalmol 2006; 51: 105-128.

16 Collewijn H, van der Mark F, Jansen TC. Precise recording of human eye movements. Vision Res 1975; 15: 447-450.

17 Kessler P, Tomlinson D, Blakeman A, Rutka J, Ranalli P, Wong AMF. The high-frequency/acceleration head heave test in detecting otolith diseases. Otol Neurotol 2007; 28: 896-904.

18 Schlenker M, Mirabella G, Goltz HC, Kessler P, Blakeman AW, Wong AMF. The linear vestibulo-ocular reflex in patients with skew deviation. Invest Ophthalmol Vis Sci 2009; 50: 168-174.

19 Goltz HC, Mirabella G, Leung JCY, Blakeman AW, Colpa L, Abuhaleeqa $\mathrm{K}$ et al. Effects of age, viewing distance and target complexity on static ocular counterroll. Vision Res 2009; 49: 1848-1852.

20 Chandrakumar M, Hirji Z, Goltz HC, Mirabella G, Blakeman AW, Colpa L et al. Effects of earth-fixed vs head-fixed targets on static ocular counterroll. Arch Ophthalmol 2010; 128: 413-417.

21 Collewijn H, Van der Steen J, Ferman L, Jansen TC. Human ocular counterroll: assessment of static and dynamic properties from electromagnetic scleral coil recordings. Exp Brain Res 1985; 59: 185-196.

22 Chandrakumar M, Blakeman A, Goltz HC, Wong AM. Static ocular counterroll in patients with skew deviation. ARVO E-Abstract 2532, 2010.

23 Parulekar MV, Dai S, Buncic JR, Wong AM. Head position-dependent changes in ocular torsion and vertical misalignment in skew deviation. Arch Ophthalmol 2008; 126: 899-905.

24 Wong AM, Colpa L, Chandrakumar M. Ability of an upright-supine test to differentiate skew deviation from other vertical strabismus causes. Arch Ophthalmol 2011; 129: 1570-1575. 